\title{
A metabonomic study of biochemical changes characteristic of genetically hypertensive rats based on ${ }^{1} \mathrm{H}$ NMR spectroscopic urinalysis
}

\author{
Kazuki Akira ${ }^{1}$, Shigenori Masu ${ }^{2}$, Misako Imachi $^{3}$, Hidemichi Mitome ${ }^{1}$ and Takao Hashimoto ${ }^{4}$
}

Spontaneously hypertensive rats (SHR) provide a simple model for studying essential hypertension. Their genetic and metabolic features are of great interest because they may provide insights into the pathophysiological processes underlying essential hypertension. We have thus investigated the metabolic characteristics of SHR at various ages, covering the prehypertensive stage and the developmental phase of hypertension, using a ${ }^{1} \mathrm{H}$ nuclear magnetic resonance (NMR)-based metabonomic approach. Twenty-four-hour urine samples from the SHR and their age-matched normotensive control, Wistar-Kyoto rats, were analyzed using ${ }^{1} \mathrm{H}$ NMR spectroscopy, and the spectral data were subjected to principal components analysis (PCA) to find metabolic differences between the two strains. Consequently, it was possible to separate the urine samples between the two strains at any age ranging from 4 to 20 weeks in the principal component scores plots. The major spectral regions and signals (metabolites) contributing to the separation were picked up based on the loadings. Subsequently, the urinary excreted levels of metabolites highlighted by the PCA were compared based on the signal intensities corrected by urine volume and body weight. These investigations revealed the major metabolic changes characteristic of the SHR, which included differences in citrate, $\alpha$-ketoglutarate, succinate, hippurate, phenylacetylglycine, $p$-cresol glucuronide, creatine, taurine, medium-chain dicarboxylates (tentative), unknown $(\delta 3.11$ ), and the regions at 3.60, 3.64, 3.68 and 3.88 p.p.m. The results supported the occurrence of metabolic acidosis in the SHR in the period of prehypertension as well as rapidly rising blood pressure. In addition, the intestinal microfloral populations in the SHR were suggested to be altered in the developmental phase of hypertension. Hypertension Research (2012) 35, 404-412; doi:10.1038/hr.2011.182; published online 17 November 2011

Keywords: metabonomics; NMR; SHR; urine

\section{INTRODUCTION}

Hypertension is a leading cause of cardiovascular morbidity and mortality. In the majority of cases, the mechanism of hypertension remains unknown because of its complex nature as a polygenic, heterogeneous and multifactorial disorder, and the disorder is classified as essential hypertension. ${ }^{1,2}$ Genetically hypertensive rat models provide a simple and useful model for studying this complex disease. ${ }^{3}$ Spontaneously hypertensive rats (SHR) are the most widely studied animal model of hypertension because some of the pathophysiological processes are similar to those of human essential hypertension. The SHR were established by inbreeding Wistar-Kyoto rats (WKY) with the highest blood pressure, ${ }^{4}$ and thus the two strains have a similar genetic background, except for the hypertension-related factors. The differences in the genetic and metabolic features between the SHR and their control normotensive WKY are expected to provide insights into blood pressure regulation in essential hypertension, ${ }^{5}$ leading to the discovery of biomarkers for the prediction and diagnosis of the disease. In addition, such animal studies are expected to facilitate the observation of hypertension-related metabolic features because the interindividual variation of genetic and metabolic factors is smaller in each strain than in humans, ${ }^{6}$ and the experiments can be carried out under the same environmental and feeding conditions.

We have thus undertaken a series of metabonomic studies using these genetically hypertensive models. In a previous paper, ${ }^{7}$ we applied ${ }^{1} \mathrm{H}$ nuclear magnetic resonance (NMR)-based metabonomic techniques to detect the metabolic differences between young SHR and the age-matched WKY. The previous study showed that several metabolites, such as citrate, $\alpha$-ketoglutarate, hippurate and creatine, were largely responsible for the separation between the two strains, illustrating the potential of such metabonomic approach in the area of hypertension research. Although the approach was promising, the study was limited to rats at 8 weeks of age. The blood pressures of the SHR and WKY rise with growth, and those of the SHR become significantly higher than those of the WKY after 5 or 6 weeks of age and steadily increase to reach systolic blood pressures of 200-250 mm Hg. ${ }^{8,9}$ Thus, it is of great interest to investigate the

${ }^{1}$ Department of Pharmaceutical Analytical Chemistry, College of Pharmaceutical Sciences, Matsuyama University, Matsuyama, Japan; ${ }^{2}$ Daisan Hospital, Jikei University School of Medicine, Tokyo, Japan; ${ }^{3}$ Bruker BioSpin KK, Tsukuba, Japan and ${ }^{4}$ School of Pharmacy, Tokyo University of Pharmacy and Life Sciences, Tokyo, Japan Correspondence: Professor K Akira, College of Pharmaceutical Sciences, Matsuyama University, 4-2 Bunkyo-cho, Matsuyama, Ehime 790-8578, Japan. E-mail: akira@cc.matsuyama-u.ac.jp

Received 29 March 2011; revised 19 September 2011; accepted 23 September 2011; published online 17 November 2011 
metabolic changes characteristic of the SHR at various ages, covering the prehypertensive stage and the developmental phase of hypertension. The hypertension- and age-related metabolic variations in plasma from the SHR and WKY have been already reported by $\mathrm{Lu}$ et al. ${ }^{10}$ using metabonomics based on gas chromatographymass spectrometry. However, they did not report any data on the developmental phase of hypertension in the SHR before 10 weeks of age. In this paper, we have characterized the urinary metabolite profiles of the SHR at ages ranging from 4 to 20 weeks using ${ }^{1} \mathrm{H}$ NMR-based metabonomics and compared them with those of age-matched WKY.

\section{MATERIALS AND METHODS}

Animals and sample collection

Six male SHR/Izm and WKY/Izm, aged 3 weeks, were obtained from the Disease Model Cooperative Research Association (Kyoto, Japan). Animal studies were conducted under approved guidelines and were reviewed by an institutional animal use committee. The animals were housed at $22{ }^{\circ} \mathrm{C}$ with a 12-hour light (0700-1900 hours) and 12-hour dark (1900-0700 hours) cycle and had free access to standard chow (CE-2, Clea Japan, Tokyo, Japan) and tap water. Rats were individually placed in metabolism cages to collect urine for $24 \mathrm{~h}$ at 4, 5, 6, 8, 10, 14 and 20 weeks of age. The volume of urine, including a small amount of washing water for the metabolism cages, was in the range of between 2 and $19 \mathrm{ml}$ depending on age and strain. Blood pressure was measured using a tail-cuff method immediately before urine collection. Other procedures concerning animal experiments and urine sample collection were the same as in the previous paper. ${ }^{7}$

\section{${ }^{1}$ H NMR spectroscopy}

Sample preparation and NMR conditions were the same as in the previous paper. ${ }^{7}$ In brief, a mixture of urine sample $(350 \mu \mathrm{l})$, phosphate-buffered solution $(\mathrm{pH} 7.4,350 \mu \mathrm{l})$ and deuterium oxide $(100 \mu \mathrm{l})$ containing sodium 3-trimethylsilyl $\left[2,2,3,3-{ }^{2} \mathrm{H}_{4}\right]$ propionate in a 5 -mm o.d. NMR tube was analyzed on a Bruker DPX400 NMR spectrometer (Bruker BioSpin, Tsukuba, Japan) at a probe temperature of $300 \mathrm{~K}$, with the water resonance suppressed using a NOESYPRESAT pulse sequence. ${ }^{11}$ Assignments of resonances were made on the basis of literature assignments ${ }^{12,13}$ and standard additions. The urinary excreted amounts of metabolites were compared based on the relative peak heights of metabolites to sodium 3-trimethylsilyl $\left[2,2,3,3-{ }^{2} \mathrm{H}_{4}\right]$ propionate, which were corrected by being multiplied by $24-\mathrm{h}$ urine volume $(\mathrm{ml})$ and divided by body weight $(\mathrm{kg})$.

\section{Data reduction of NMR data and principal component analysis}

All NMR spectra were phased and baseline corrected. Data reduction of NMR data and principal components analysis (PCA) were performed using AMIX software (version 3.6.8; Bruker BioSpin). The NMR spectra ( $\delta$ 0.5-9.5) were automatically reduced to 225 segmented regions of equal width (0.04 p.p.m.). The region between $\delta 4.3$ and $\delta 6.5$ was removed before PCA to eliminate the effects of the variation in the suppression of the water resonance and in the urea signal caused by partial ${ }^{1} \mathrm{H}$ exchange by deuterium oxide. Following the removal of the region, the integral intensities of the segmented regions in a spectrum were divided by that of creatinine $(\delta 4.047-4.067)$. This procedure normalized each spectral data set to compensate for variations in urine volumes. The normalized data sets from the spectra ( 170 variables) were then subjected to PCA after mean-centering. Scores plots of the principal components (PCs) were constructed to visualize the separation of the urine samples between the groups. The loadings of individual variables (segmented regions) were obtained, and the regions and peaks (metabolites) contributed to the separation between the groups were identified based on the loadings for the PCs, which represented the separation. The loadings indicate the importance of the regions for the separation and the metabolic differences between the groups.

\section{Statistical significance testing}

Data are expressed as the mean \pm s.d. Statistical analysis was done using an unpaired $t$-test. A $P$-value of $<0.05$ was considered statistically significant.

\section{RESULTS}

The body weights and blood pressures of the rats are summarized in Table 1. The body weights of the SHR after 10 weeks of age were significantly lower than those of the age-matched WKY. The systolic and diastolic blood pressures of the SHR after 5 weeks of age were significantly higher than those of the age-matched WKY. Urine samples were obtained from the SHR and WKY at various ages up to 20 weeks of age, followed by ${ }^{1} \mathrm{H}$ NMR urinalysis. The SHR excreted less urine than the WKY, and the propensity was prominent in the aged rats. Typical spectra of rat urine at 5 weeks of age are illustrated in Figure 1. Although variations in citrate signal intensities were obvious between the two strains at various ages, further visual comparison was difficult. Thus, to fully characterize the metabolic differences among the data sets, an unsupervised chemometric method, PCA, was employed.

\section{PC scores plots and loadings}

PCA was separately performed for each age to compare the urinary metabolic profiles between the SHR and WKY. The PC1 vs. PC2 scores plots showed a clear separation of urine samples between the two strains at any age, as shown in Figure 2. These results indicate that there were metabolic changes between the two strains over the period examined. The most obvious separation was seen at 8 weeks of age, showing the largest strain-related metabolic difference among various ages. The samples from the hypertensive and normotensive strains at $4,5,8$ and 10 weeks of age were separated along the first PC in the scores plots, whereas at 6,14 and 20 weeks of age they were obliquely separated. In order to determine the spectral regions and metabolites contributing to the separation on the PCA maps, the PC1 loadings were examined at $4,5,8$ and 10 weeks of age. Consequently, most 0.04 p.p.m. regions showed positive PC1 loadings at the four separate age groups, whereas the samples from the SHR had lower PC1 scores, as shown in Figure 2. These results showed that the integral intensities of most regions were smaller in the SHR than in the WKY, and most

\section{Table 1 Characteristics of the SHR and WKYa}

\begin{tabular}{|c|c|c|c|c|}
\hline \multirow[b]{2}{*}{ Age (weeks) } & \multirow[b]{2}{*}{ Strain } & \multirow[b]{2}{*}{ Body weight (g) } & \multicolumn{2}{|c|}{$B P(m m H g)$} \\
\hline & & & $S B P$ & $D B P$ \\
\hline \multirow[t]{2}{*}{4} & SHR & $62 \pm 2$ & $88 \pm 32$ & $63 \pm 20$ \\
\hline & WKY & $64 \pm 5$ & $80 \pm 12$ & $57 \pm 7$ \\
\hline \multirow[t]{2}{*}{5} & SHR & $98 \pm 5$ & $131 \pm 8^{*}$ & $96 \pm 8^{*}$ \\
\hline & WKY & $96 \pm 8$ & $115 \pm 4$ & $85 \pm 6$ \\
\hline \multirow[t]{2}{*}{6} & SHR & $133 \pm 6$ & $148 \pm 7^{*}$ & $106 \pm 7 *$ \\
\hline & WKY & $129 \pm 12$ & $113 \pm 8$ & $78 \pm 5$ \\
\hline \multirow[t]{2}{*}{8} & SHR & $205 \pm 8$ & $157 \pm 10^{*}$ & $113 \pm 11$ * \\
\hline & WKY & $207 \pm 14$ & $122 \pm 5$ & $78 \pm 6$ \\
\hline \multirow[t]{2}{*}{10} & SHR & $246 \pm 11^{*}$ & $182 \pm 8^{*}$ & $128 \pm 8^{*}$ \\
\hline & WKY & $272 \pm 16$ & $130 \pm 6$ & $87 \pm 10$ \\
\hline \multirow[t]{2}{*}{14} & SHR & $301 \pm 18^{*}$ & $205 \pm 8^{*}$ & $153 \pm 9 *$ \\
\hline & WKY & $348 \pm 17$ & $134 \pm 5$ & $103 \pm 7$ \\
\hline \multirow[t]{2}{*}{20} & SHR & $335 \pm 13^{*}$ & $207 \pm 18^{*}$ & $155 \pm 10$ * \\
\hline & WKY & $390 \pm 14$ & $139 \pm 8$ & $104 \pm 8$ \\
\hline
\end{tabular}

Abbreviations: BP, blood pressure; DBP, diastolic BP; SBP, systolic BP; SHR, spontaneously hypertensive rats; WKY, Wistar-Kyoto rats.

hypertensive rats; WKY,
aValues are mean \pm s.d. Values are mean \pm
$* P<0.05$ vs. WKY. 

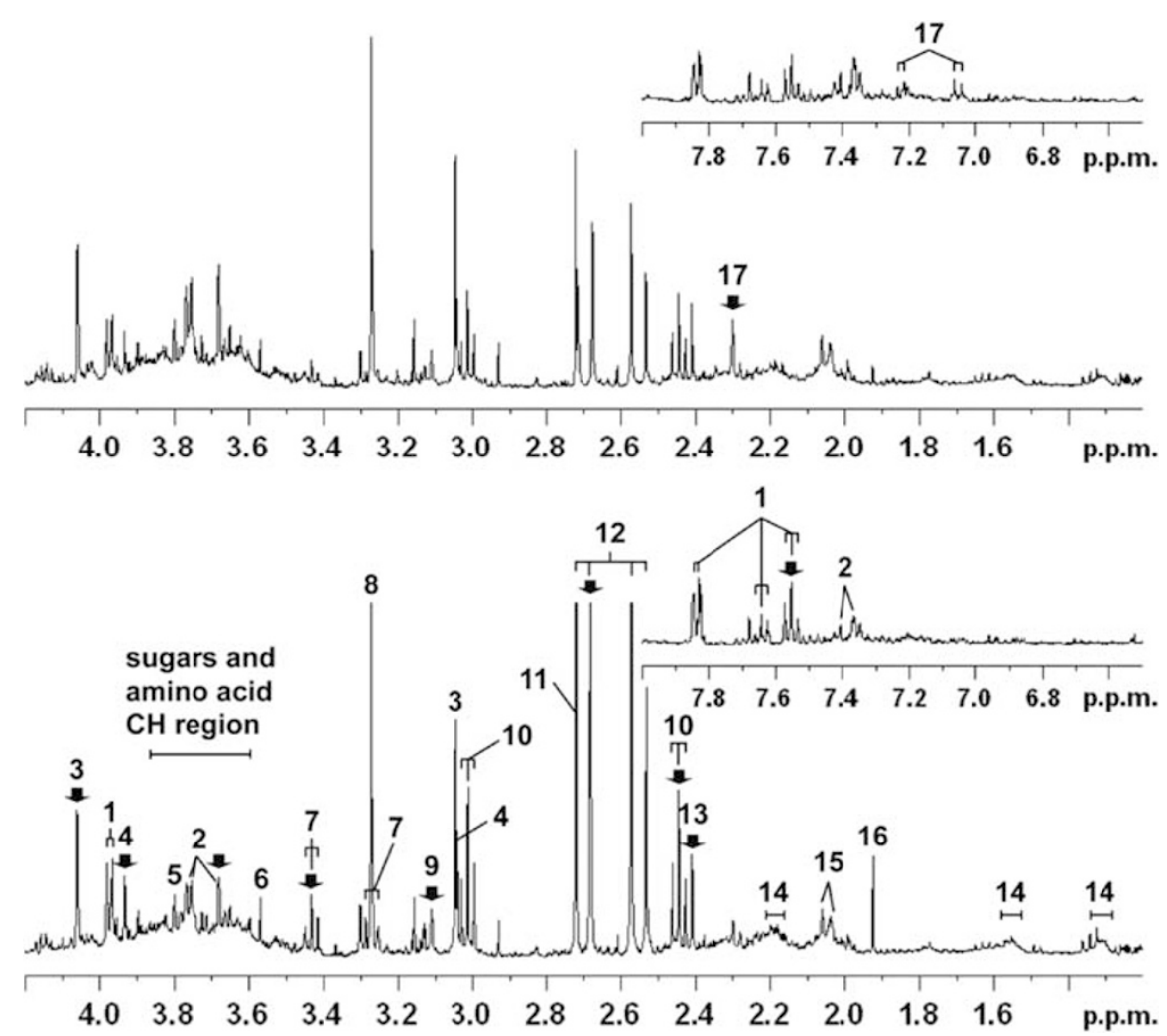

Figure 1 Typical ${ }^{1} \mathrm{H}$ nuclear magnetic resonance spectra obtained from urine samples of rats aged 5 weeks. The upper and lower spectra were obtained from the spontaneously hypertensive rats and Wistar-Kyoto rats, respectively. The spectra have been scaled so that the intensity of the methylene peak (peak number 3 ) due to creatinine is the same. Key to numbered peaks: 1 , hippurate; 2 , phenylacetylglycine; 3 , creatinine; 4 , creatine; 5 , guanidinoacetate; 6 , glycine; 7, taurine; 8, trimethylamine $\mathrm{N}$-oxide; 9, unknown; 10, $\alpha$-ketoglutarate; 11, dimethylamine; 12, citrate; 13, succinate; 14, medium-chain dicarboxylates (tentative); 15, $\mathrm{N}$-acetyls of glycoproteins; 16 , acetate; and 17, $\mathrm{p}$-cresol glucuronide. Urinary excreted amounts of metabolites were evaluated using the peaks indicated by the arrows.
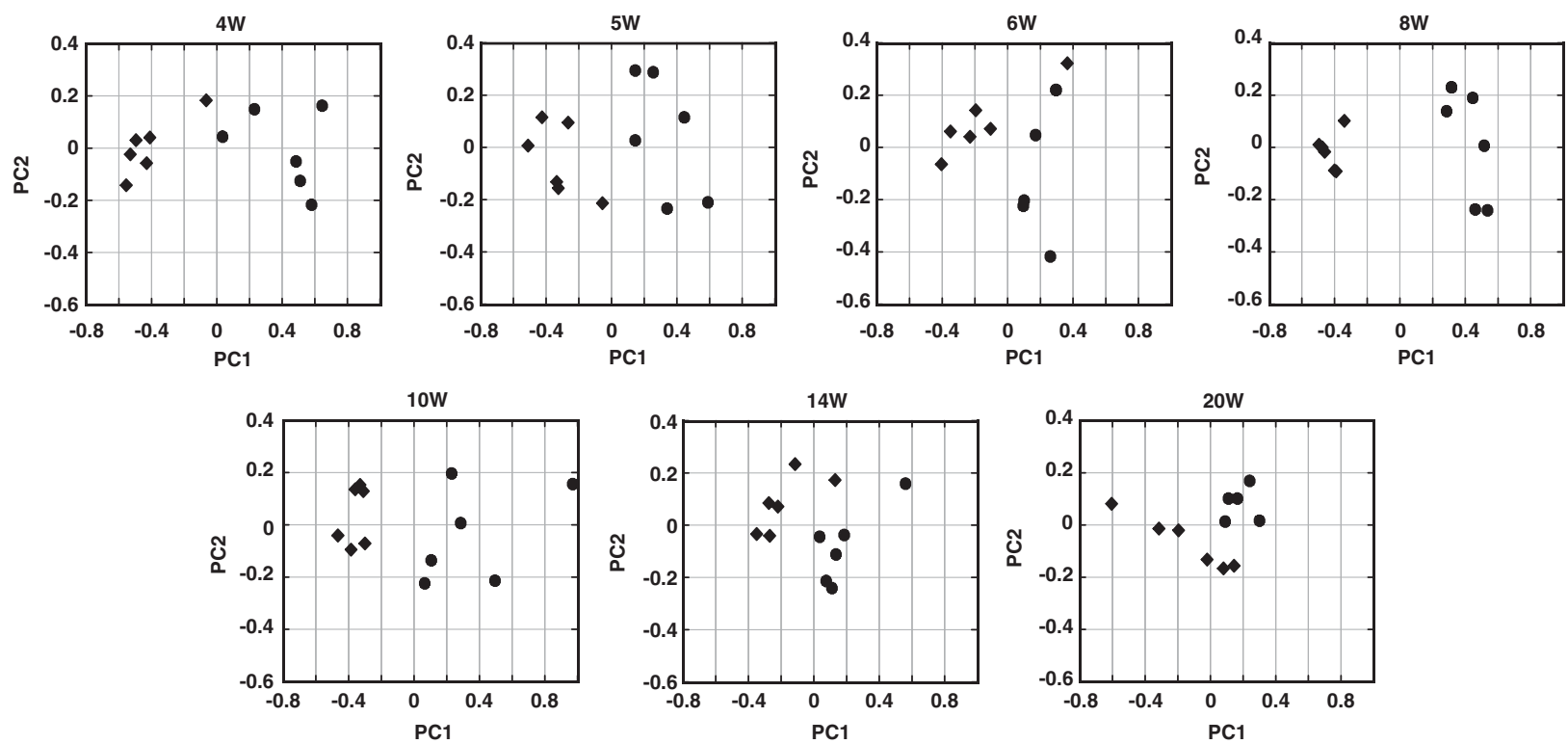

Figure 2 Principal component 1 (PC1)-PC2 scores plots derived from the ${ }^{1} \mathrm{H}$ nuclear magnetic resonance spectra of urine samples of the spontaneously hypertensive rats (SHR) and Wistar-Kyoto rats (WKY). Key: filled lozenge, SHR; filled circle, WKY. The two PCs captured 77-90\% of the variation (50-80\% for PC1). One spectrum from the 6-week-old WKY was eliminated from the calculation because no satisfactory phase correction was obtained for the spectrum probably owing to a slight deviation of the irradiation frequency for water suppression. One of the WKY is missing from the experiments of the 20-week-old rats due to death. 
urinary metabolites in the former were excreted in $24 \mathrm{~h}$ less than in the latter, based on the urinary levels of creatinine. In this case, the larger positive $\mathrm{PC} 1$ loadings mean more significant decreases in the excretion of the metabolites of the regions in the SHR. The major spectral regions contributing to the separation between the SHR and WKY are summarized in Table 2, with the assignment of the pronounced signals contained in those regions.
Common metabolic changes in various ages

By inspection of the PC loadings together with the ${ }^{1} \mathrm{H}$ NMR spectra of the urine samples, the most distinctive metabolic changes in the SHR, which were common thorough the period examined, were decreases in urinary levels of citrate, $\alpha$-ketoglutarate, hippurate and the integral intensities of the regions at 2.20, 2.24, 2.40 and 3.12 p.p.m. The region at 2.20 p.p.m. was considered to contain signals due to methylene

Table 2 Summary of major metabolic differences between the SHR and WKY observed in the PCA of ${ }^{1} \mathbf{H}$ NMR spectral data ${ }^{a}$

\begin{tabular}{|c|c|c|c|c|c|}
\hline \multirow{2}{*}{$\begin{array}{l}\text { Integrated } \\
\text { Spectral region }\end{array}$} & \multicolumn{4}{|c|}{ Increase or decrease in the $S H R^{\mathrm{b}}$} & \multirow[b]{2}{*}{ Major metabolite(s) present in selected region ( $\delta$, multiplicity) } \\
\hline & Week 4 & Week 5 & Week 8 & Week 10 & \\
\hline 7.84 & $\downarrow$ & $\downarrow$ & $\downarrow \downarrow$ & $\downarrow \downarrow$ & Hippurate $(7.84, \mathrm{~d})$ \\
\hline 7.64 & & & $\downarrow$ & $\downarrow \downarrow$ & Hippurate $(7.65, \mathrm{t})$ \\
\hline 7.56 & $\downarrow$ & $\downarrow$ & $\downarrow \downarrow$ & $\downarrow \downarrow$ & Hippurate $(7.56, \mathrm{t})$ \\
\hline 7.52 & & & & $\downarrow$ & Hippurate $(7.56, \mathrm{t})$ \\
\hline $7.36^{\mathrm{d}}$ & $\downarrow$ & $\uparrow$ & -0.030 & -0.036 & Phenylacetylglycine $(7.35-7.38, \mathrm{~m})$ \\
\hline 4.00 & & & $\downarrow$ & $\downarrow$ & Hippurate $(3.97, \mathrm{~d})$ \\
\hline 3.96 & $\downarrow$ & $\downarrow$ & $\downarrow \downarrow$ & $\downarrow \downarrow$ & Hippurate $(3.97$, d) \\
\hline $3.88^{d}$ & $\downarrow$ & -0.012 & $\downarrow$ & $\downarrow$ & e \\
\hline 3.80 & $\downarrow$ & & $\downarrow \downarrow$ & $\downarrow$ & Guanidinoacetate $(3.80, \mathrm{~s})$ \\
\hline $3.76^{d}$ & $\downarrow$ & $\uparrow \uparrow$ & -0.019 & -0.038 & Phenylacetylglycine $(3.76, \mathrm{~d})$ \\
\hline 3.72 & $\downarrow$ & & $\downarrow$ & & e \\
\hline $3.68^{d}$ & $\downarrow \downarrow$ & -0.036 & 0.044 & 0.013 & Phenylacetylglycine $(3.68, \mathrm{~s})$ \\
\hline $3.64^{d}$ & $\downarrow$ & -0.040 & 0.049 & 0.014 & e \\
\hline $3.60^{d}$ & & -0.013 & $\downarrow$ & $\downarrow$ & e \\
\hline 3.56 & & & $\downarrow$ & $\downarrow$ & Glycine $(3.57, \mathrm{~s})$ \\
\hline $3.44^{d}$ & & $\downarrow \downarrow$ & $\downarrow \downarrow$ & -0.020 & Taurine $(3.43, \mathrm{t})$ \\
\hline 3.32 & & & $\downarrow$ & $\downarrow$ & e \\
\hline 3.28 & $\downarrow \downarrow$ & & $\downarrow \downarrow$ & $\downarrow$ & $\operatorname{TMAO}(3.27, \mathrm{~s})$, taurine $(3.27, \mathrm{t})$ \\
\hline 3.24 & & & $\downarrow$ & & e \\
\hline 3.16 & & & $\downarrow$ & & e \\
\hline 3.12 & $\downarrow$ & $\downarrow$ & $\downarrow$ & $\downarrow \downarrow$ & e \\
\hline 3.00 & $\downarrow \downarrow$ & $\downarrow \downarrow$ & $\downarrow \downarrow$ & $\downarrow \downarrow$ & $\alpha$-Ketoglutarate $(3.01, \mathrm{t})$ \\
\hline 2.72 & $\downarrow \downarrow$ & $\downarrow \downarrow$ & $\downarrow \downarrow$ & $\downarrow \downarrow$ & Citrate $(2.69, \mathrm{~d}), \mathrm{DMA}(2.72, \mathrm{~s})$ \\
\hline 2.68 & $\downarrow \downarrow$ & $\downarrow \downarrow$ & $\downarrow \downarrow$ & $\downarrow \downarrow$ & Citrate $(2.69, \mathrm{~d})$ \\
\hline 2.60 & $\downarrow$ & $\downarrow$ & $\downarrow \downarrow$ & $\downarrow \downarrow$ & Citrate $(2.55, \mathrm{~d})$ \\
\hline $2.56,2.52$ & $\downarrow \downarrow$ & $\downarrow \downarrow$ & $\downarrow \downarrow$ & $\downarrow \downarrow$ & Citrate $(2.55, \mathrm{~d})$ \\
\hline 2.48 & $\downarrow$ & $\downarrow$ & $\downarrow \downarrow$ & $\downarrow \downarrow$ & $\alpha$-Ketoglutarate $(2.45, \mathrm{t})$ \\
\hline 2.44 & $\downarrow \downarrow$ & $\downarrow \downarrow$ & $\downarrow \downarrow$ & $\downarrow \downarrow$ & $\alpha$-Ketoglutarate $(2.45, \mathrm{t})$ \\
\hline 2.40 & $\downarrow$ & $\downarrow$ & $\downarrow$ & $\downarrow$ & Succinate $(2.41, \mathrm{~s})$ \\
\hline $2.32^{\mathrm{d}}$ & $\downarrow$ & -0.009 & 0.032 & -0.005 & p-Cresol glucuronide \\
\hline 2.24 & $\downarrow$ & $\downarrow$ & $\downarrow$ & $\downarrow$ & e \\
\hline 2.20 & $\downarrow \downarrow$ & $\downarrow \downarrow$ & $\downarrow$ & $\downarrow$ & MCDAs \\
\hline 2.16 & $\downarrow$ & $\downarrow$ & $\downarrow$ & & MCDAs \\
\hline 2.04 & & & $\downarrow$ & $\downarrow$ & $\begin{array}{l}N \text {-acetyls of glycoproteins } \\
(2.03-2.07)\end{array}$ \\
\hline 2.00 & & & $\downarrow$ & $\downarrow$ & e \\
\hline 1.92 & $\downarrow \downarrow$ & & $\downarrow$ & & Acetate $(1.92, \mathrm{~s})$ \\
\hline 1.60 & & $\downarrow$ & & & e \\
\hline 1.56 & $\downarrow \downarrow$ & $\downarrow \downarrow$ & $\downarrow$ & 0.047 & MCDAs \\
\hline 1.52 & $\downarrow$ & $\downarrow$ & & & MCDAs \\
\hline 1.36 & & $\downarrow$ & & & e \\
\hline 1.32 & $\downarrow$ & $\downarrow \downarrow$ & 0.042 & 0.028 & MCDAs \\
\hline 1.28 & $\downarrow$ & $\downarrow$ & & & MCDAs \\
\hline
\end{tabular}

Abbreviations: DMA, dimethylamine; MCDAs, medium-chain dicarboxylates (tentatively identified); NMR, nuclear magnetic resonance; PC, principal components; PCA, principal components analysis; SHR, spontaneously hypertensive rats; TMAO, trimethylamine $N$-oxide; WKY, Wistar-Kyoto rats.

aUrine samples from the SHR and WKY were analyzed by ${ }^{1} \mathrm{H}$ NMR spectroscopy, followed by PCA. This table shows the major metabolic differences between the two strains.

bThe upward and downward arrows represent the increases and decreases in the integral intensities of the regions, respectively. PC1 loadings: $\uparrow,>-0.1$ and $=<-0.05 ; \uparrow \uparrow,=<-0.1 ; \downarrow$, $=>0.05$ and $<0.1$; and $\downarrow \downarrow,=>0.1$. The loadings between -0.05 and 0.05 are, if necessary, indicated.

cThe buckets contributing to the discrimination between the strains on the PCA maps were selected based on their PC1 loadings. The middle $\delta$-value of each region was indicated. dRegions with large age-related changes in loadings.

eUnidentified. 
protons of medium-chain dicarboxylates such as suberate, ${ }^{14}$ and the intensity of the region varied with those of the regions at 1.56 and 1.32 p.p.m., which contained multiplet signals due to other methylene protons of the medium-chain dicarboxylates. The region at 2.40 p.p.m. contained a singlet signal due to succinate at $\delta 2.41$. The region at 3.12 p.p.m. contained a singlet signal at $\delta 3.11$ due to an unknown metabolite. The change of the region at 3.28 p.p.m. was also prominent at 4,8 and 10 weeks of age. Trimethylamine $N$-oxide was detected as a major singlet signal at $\delta 3.27$, and frequently broadened owing to overlap with signals due to taurine and betaine. ${ }^{15,16}$ The extent of overlap of taurine to this signal could be estimated from another signal of taurine at $\delta 3.43$. Although the presence of betaine, which gives another singlet signal at $\delta 3.90$, was ambiguous, trimethylamine $\mathrm{N}$-oxide was thought to be excreted more in younger rats than in older rats, ${ }^{17}$ and less in the SHR than in the WKY after 8 weeks of age, based on the creatinine signal intensity.

\section{Age-related metabolic changes characteristic of the SHR}

The age-related metabolic changes characteristic of the SHR are also of interest. Thus, the variables whose loadings largely changed with age should be noted in the above PCA for each age. Such variables were the regions at $2.32,3.44,3.60,3.64,3.68,3.76,3.88$ and 7.36 p.p.m. in Table 2. The loadings showed that the metabolites in the spectral regions at 2.32, 3.60, 3.64, 3.68 and 3.88 p.p.m. were less excreted in the SHR than in the WKY at 4 weeks of age, whereas at 5 weeks of age they were more excreted in the SHR, and then the relationship returned to the original state at 8 weeks of age. The region at 2.32 p.p.m. partially included a singlet signal due to the methyl group of $p$-cresol glucuronide ${ }^{18,19}$ at $\delta 2.30$. The major metabolites contributing to the regions at $3.60,3.64,3.68$ and 3.88 p.p.m. were unknown. The region at 3.68 p.p.m. also reflected the level of phenylacetylglycine. The loading showed that the metabolites in the region at 3.44 p.p.m. were excreted in lesser quantities in the SHR at 5 and 8 weeks of age, and in greater quantities in the SHR at 10 weeks of age. These changes in the region at 3.44 p.p.m. were obviously due to taurine, as it appeared as a major signal in the region after 5 weeks of age. Interestingly, appreciable signals due to taurine were unobserved at 4 weeks of age. The loadings for the regions at 3.76 and 7.36 p.p.m. showed pronounced changes from 4 to 5 weeks of age. These regions contained prominent resonances due to phenylacetylglycine. In the spectra of 14- and 20-week-old WKY, the signals due to succinyltaurine, recently identified by us, ${ }^{20}$ were clearly detected at around $\delta 2.5,3.1$ and 3.6. It was demonstrated here again that this metabolite was not observed in urine from the SHR and was characteristic of the aged WKY.

\section{Metabolic changes based on peak intensities}

In order to make the metabolic changes picked up by the PCA clearer, the excreted levels of metabolites were compared between the SHR and WKY on the basis of the peak intensities corrected, as shown in the method section. Consequently, the following results were obtained as illustrated in Figure 3.

Decreases in citrate, $\alpha$-ketoglutarate and hippurate in the SHR at 4 , 5,8 and 10 weeks of age, which were the observations in Table 2, were confirmed, except the hippurate level at 4 weeks of age. The propensity of decrease in the citrate and $\alpha$-ketoglutarate levels was also seen in the SHR at 14 and 20 weeks of age. On the contrary, at 6 weeks of age, no significant difference was seen between the strains in the excretion of citrate, $\alpha$-ketoglutarate and hippurate. Another tricarboxylic acid cycle (TCA) cycle intermediate, succinate, was found to be less excreted in the SHR than in the WKY at 8 and 10 weeks of age. The excreted amount of creatinine was also compared, which was impossible in the PCA based on creatinine normalization. The excreted level of creatinine was 1.3 times more in the SHR than in the WKY at 4 weeks of age, whereas at other ages no significant difference in creatinine output was seen between the two strains. Thus, in the present PCA analysis, based on creatinine intensity, the excretion of all metabolites in the SHR at 4 weeks of age was, as a whole, found to be underestimated. Thus, the results at 4 weeks of age in Table 2 are partially misleading. This underestimation is the reason for the above contradiction of the hippurate level at 4 weeks of age.

The SHR had a propensity to excrete less taurine than the WKY from ages 5 to 8 weeks, and the decrease was significant at 6 and 8 weeks of age. In contrast, no significant change was observed at 14 weeks of age, and at 20 weeks of age the metabolite was excreted more in the SHR. In the previous metabonomic study, ${ }^{21}$ stroke-prone spontaneously hypertensive rats with relatively old ages, that is, 12 and 26 weeks of age, were shown to excrete more taurine than the age-matched WKY, although no examination was performed on the younger rats. The stroke-prone spontaneously hypertensive rats are a substrain derived from the SHR, and often used as another hypertensive model because of more severe hypertension. Although the age of hypertaurinuria was different between the two hypertensive models, there might be the common propensity to excrete more taurine in older hypertensive rats. The excreted level of the unknown metabolite at $\delta 3.11$ was found to be significantly decreased in the SHR than in the WKY at ages 5-14 weeks. Phenylacetylglycine was found to be more excreted in the SHR at 5 weeks of age, whereas at 6 weeks of age the metabolite was less excreted in the SHR, and the differences were significant. These results of taurine, phenylacetylglycine and unknown in the developmental phase of hypertension, except for 4 weeks of age, were consistent with those in Table 2. p-Cresol glucuronide had an exceptional propensity to be excreted more in the SHR than in the WKY at ages other than 6 weeks, and the increased excretions at 5 and 14 weeks of age were statistically significant. In addition, the output of creatine, which was not noted in Table 2, was found to be prominent in younger rats, with a large interindividual variation, and the increased excretion in the SHR at 4 weeks of age was significant. Although the output of creatine decreased after 8 weeks of age, and appreciable signals, due to creatine, were frequently unobserved particularly in the WKY, the SHR still had a propensity to excrete the metabolite more than the WKY. At 8 weeks of age, appreciable signals due to creatine were observed in two of the six SHR, whereas in the WKY no appreciable signals were detected. Similar results were also obtained in a previous study of 8 -week-old rats. ${ }^{7}$

The relations of the urinary outputs of the above metabolites were examined to understand more about the observed metabolic changes. Consequently, high correlation coefficients were observed among the three TCA cycle intermediates and between phenylacetylglycine and $p$-cresol glucuronide (see below). In particular, there was a very high correlation between citrate and $\alpha$-ketoglutarate, as shown in Figure 4.

The data in Figure 3 also show the age-related metabolic changes of various metabolite levels, although the aging effects themselves are not the subject of this study. A general decrease in the excreted levels of the metabolites, other than taurine and creatinine, was observed after 5 or 6 weeks of age in both strains. In contrast, increases with aging were observed in creatinine for both strains, and in taurine for the SHR only. ${ }^{17,22}$ 

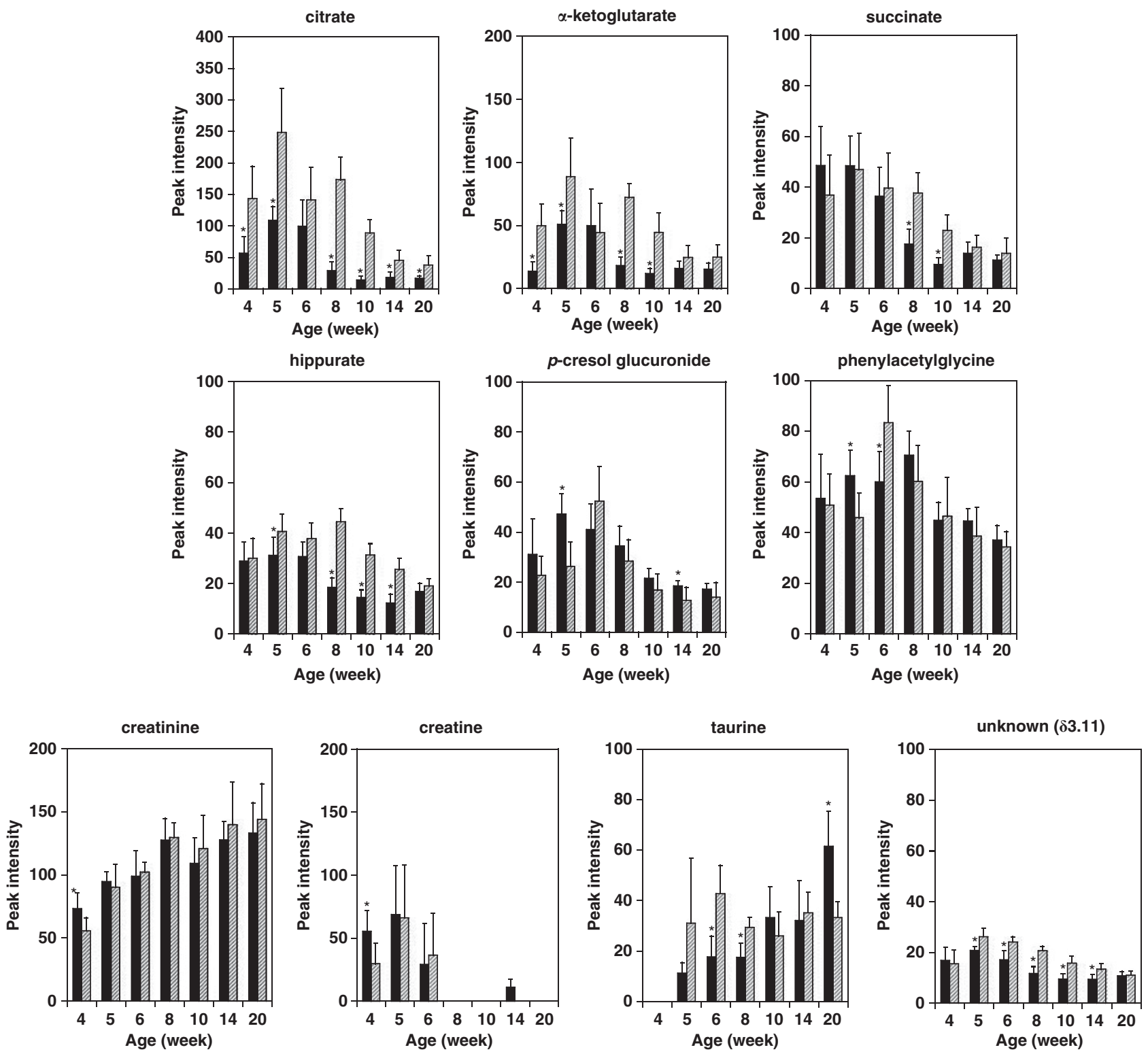

Figure 3 Comparison of urinary excreted amounts of metabolites noted in the principal components analysis between the spontaneously hypertensive rats (SHR) and Wistar-Kyoto rats (WKY). The axis of ordinates indicates the relative peak intensities of metabolites, corrected as shown in the experimental section. Key: black bars, SHR; shaded bars, WKY. The data from one 6-week-old WKY were eliminated owing to the reason described in Figure 2. The data of creatine in the WKY at 4 weeks of age were obtained from five rats because one spectrum showed no peak due to the metabolite. Data expressed as mean \pm s.d. ${ }^{*} P<0.05$ vs. WKY.

\section{DISCUSSION}

Before PCA of NMR spectral data, an adequate normalization for the data is indispensable to remove or minimize the effects of variable dilution of the urine samples. ${ }^{23}$ Total integral normalization is generally used for NMR-based metabonomics, assuming that the total amounts of metabolites in urine samples and the total integral intensities of ${ }^{1} \mathrm{H}$ NMR signals are almost constant, irrespective of gene function as well as pathophysiological and toxicological events. However, these assumptions are not always the case in practice, for example, when renal handling of metabolites significantly changes and some spectral regions containing major metabolite signals are removed before PCA. The choice of normalization method is particularly important when an increase or decrease in urinary output of metabolites is being discussed. In our study, creatinine normalization was used because creatinine is generally accepted as a marker of urine dilution. The creatinine normalization was adequate for the ages from 5 to 20 weeks because there was no significant difference in the urinary excreted amount of creatinine between the SHR and WKY.

In this study, the spectral regions and peaks (metabolites) contributing to the separation between the SHR and WKY were picked up by PCA and subsequently the excreted amounts of selected metabolites were quantitatively compared based on the peak intensities. In the previous paper, ${ }^{7}$ a metabonomic study of urine samples from 8-weekold SHR and WKY was performed. The metabolic changes observed for the 8-week-old rats in the present study were substantially consistent with those in the previous one.

The most significant metabolites distinguishing between the strains were citrate and $\alpha$-ketoglutarate in younger rats, except at 6 weeks of 


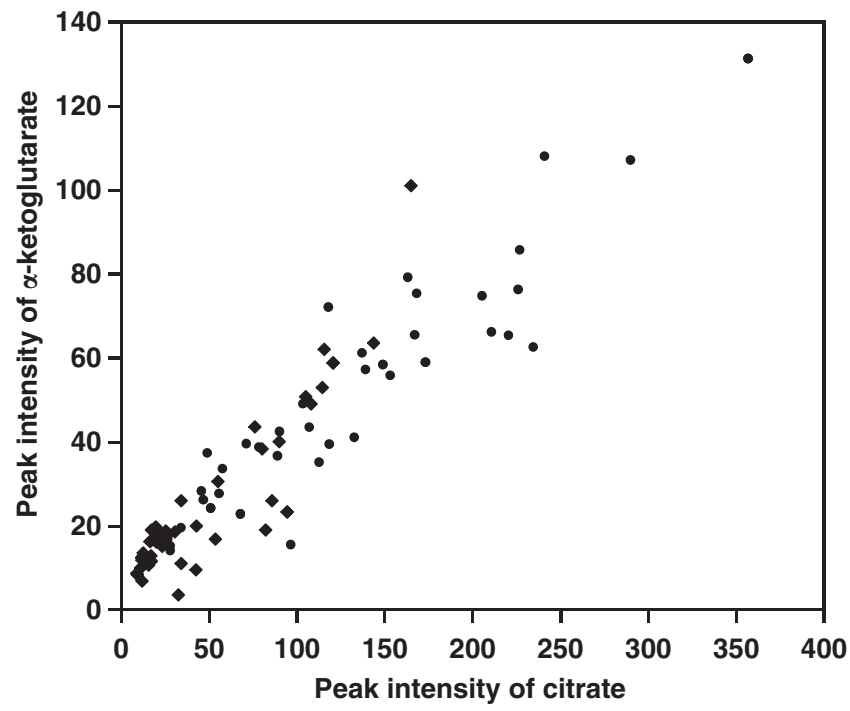

Figure 4 Relationship between the urinary excreted amounts of citrate and $\alpha$-ketoglutarate. The axes indicate the relative peak intensities of metabolites corrected, as shown in the method section. The correlation coefficient was 0.936 for all data points, and those for the data points from the spontaneously hypertensive rats (SHR) and Wistar-Kyoto rats (WKY) were 0.909 and 0.933 , respectively. Key: filled lozenge, SHR; filled circle, WKY.

age. Citrate is one of the TCA cycle intermediates and has a central role in energy metabolism. The metabolite has been previously reported to be excreted in urine much in smaller amounts in the SHR aged 11-15 weeks than in the age-matched WKY. ${ }^{24}$ The decreased excretion of citrate was also reported in the SHR at 8 weeks of age in our previous work. ${ }^{7}$ Recent reports have shown that urinary citrate excretion decreases in hypertensive patients. ${ }^{25,26}$ The decreased citrate excretion has been explained to be due to increased proximal tubular citrate reabsorption resulting from metabolic acidosis. ${ }^{27}$ Indeed, the SHR at 6-12 weeks of age are mildly acidotic. ${ }^{28}$ Sharma et al. ${ }^{29}$ have reported the presence of mild metabolic acidosis in young normotensive salt-sensitive human. The mechanisms of the increased citrate reabsorption associated with chronic metabolic acidosis in rats have been investigated in many papers. One mechanism is the increase in transported bivalent citrate anion by reduced $\mathrm{pH}$ in tubular lumen. ${ }^{30}$ The bivalent citrate anion is incorporated into tubular cells through an apical membrane $\mathrm{Na}$ /dicarboxylate co-transporter, ${ }^{31}$ which also accepts other TCA cycle intermediates. The other mechanism is an intracellular decrease in citrate levels. Such a decrease is caused by the enhanced activity of ATP citrate lyase in the cytosol, which catalyzes the conversion of citrate and coenzyme A to oxaloacetate and acetyl coenzyme A. ${ }^{27}$ Decreased activity of citrate synthetase in the mitochondrial TCA cycle is another reason for the decreased intracellular citrate levels. ${ }^{32}$ In addition, the increased activity of aconitate hydratase, the first step in mitochondrial citrate metabolism, has been reported to contribute to the intracellular decrease in citrate levels. $^{33}$

The observed hypocitraturia in the SHR at 4 and 5 weeks of age implies the occurrence of metabolic acidosis before the development of hypertension, suggesting that the acidosis is not the result of elevated blood pressure. ${ }^{28}$ Additional research is required to elucidate the relationship between hypertension and the deranged kidney metabolism as well as the metabolic acidosis, ${ }^{25}$ as there is a hypothesis that the kidney has a major role in the development of hypertension. ${ }^{34}$ It is noteworthy that no significant differences in the urinary levels of citrate and $\alpha$-ketoglutarate were observed between the two strains at 6 weeks of age, whereas before and after the age the differences were significant. It seems that the metabolic changes from 5 to 6 weeks of age were different between the two strains; in the SHR these metabolite levels were almost constant in both ages, whereas in the WKY they were considerably decreased. The significance of these differences in metabolic changes, with the underlying gene and protein expressions, is the subject of further investigation.

The observed decrease in the urinary output of another TCA cycle intermediate, $\alpha$-ketoglutarate, is considered to be also due to the enhanced tubular reabsorption resulting from the cellular depletion of the metabolite, whose formation is presumed to be reduced by the depletion of the precursor, citrate. As can be seen in Figure 3, citrate and $\alpha$-ketoglutarate closely resembled each other in their bar graph patterns for both strains. The urinary levels of the two metabolites showed a very high correlation, as shown in Figure 4. This observation seems to reflect the correlation between the depleted levels of citrate and $\alpha$-ketoglutarate in renal cells, and supports the above presumption that cellular citrate depletion causes a decrease in cellular $\alpha$-ketoglutarate level. There were also high correlations between citrate and succinate $(r=0.720)$, and $\alpha$-ketoglutarate and succinate $(r=0.627)$. Therefore, the decreased excretion of succinate at 8 and 10 weeks of age in the SHR may be due to the cellular depletion of citrate and $\alpha$-ketoglutarate, which are the precursors for succinate. Nissim et al. ${ }^{32}$ have reported a significantly higher flux through $\alpha$-ketoglutarate, succinate, malate and glutamate dehydrogenase, and through glutaminase in acidotic rats. The increased activities of the TCA cycle enzymes decrease the $\alpha$-ketoglutarate levels, whereas the depleted $\alpha$-ketoglutarate pool is preserved by the increased activities of glutaminase and glutamate dehydrogenase, which catalyze the formation of $\alpha$-ketoglutarate from glutamine. Thus, the effects of these enzymatic alterations on the cellular levels of $\alpha$-ketoglutarate and succinate are unclear.

The difference in hippurate excretion between the two strains was remarkable at ages from 5 to 14 weeks, except for 6 weeks. This metabolite is readily formed in the body from benzoate, which originates from the action of intestinal microflora on dietary aromatic compounds. ${ }^{35}$ Therefore, the present results probably reflect the differences in the intestinal microfloral populations and their metabolisms. Holmes et al. ${ }^{36}$ have studied the association of metabolic profiles with diet and blood pressure in humans, and reported an inverse association of urinary hippurate levels with blood pressure. This observation is consistent with the present results where the hypertensive strain excreted less hippurate than the normotensive strain. It should be noted that in our study no decrease in urinary excretion of the metabolite was observed at the prehypertensive phase, namely, 4 weeks of age. The above study in humans indicated the possible association of diet to the altered intestinal microflora. On the contrary, the present study suggests that some genetic and metabolic factors characteristic of the SHR were the cause of the altered microflora because the host and intestinal microbes interact through the exchange and co-metabolism of substrates, ${ }^{37}$ and diet was common for the two strains. Hypertension may be associated with some genetic and metabolic factors, regulating the formation of the intestinal microflora that produces less benzoate.

Phenylacetylglycine and $p$-cresol glucuronide are generated from phenylacetate and $p$-cresol, respectively, via phase II detoxification mechanism in the liver or the gut mucosa. Phenylacetate and $p$-cresol are also reported to originate from intestinal microfloral metabolism. ${ }^{18,35}$ The metabolic changes of the two conjugates between the SHR and WKY, observed at 5 and 6 weeks of age, were somewhat 
strange. Interestingly, the bar graph patterns of these metabolites resembled each other at the two ages. High correlation coefficients were obtained between phenylacetylglycine and $p$-cresol glucuronide levels ( $r=0.849$ for all ages, $r=0.842$ for 5 and 6 weeks of age). On the contrary, there were no correlations between hippurate and these metabolites. Therefore, it seems likely that phenylacetate and $p$-cresol are formed by the same intestinal microbes, ${ }^{18}$ which are different from the microbes responsible for the formation of benzoate, a precursor of hippurate. Thus, this observation indicates that the activities of microbes responsible for the formation of phenylacetate and $p$-cresol are different between the SHR and WKY during the limited period.

In the previous paper, ${ }^{7}$ the metabolites in the 7.36 p.p.m. region, which substantially corresponded to phenylacetylglycine, were less excreted in the SHR at 8 weeks of age, whereas in the present study the metabolite was shown to be more excreted in the SHR as shown in Table 2, although the increase was not significant (Figure 3). The intestinal microflora is affected by breeding conditions, including diet, after birth. The ages, when the rats were carried in the breeding facilities of the university, were 3 and 7 weeks after birth in the present and previous studies, respectively. Thus, it is possible that the microflora responsible for the production of phenylacetate was differently formed during the breeding before 8 weeks of age in the present study.

Taurine is the most abundant free amino acid having beneficial effects in mammalian tissues. Taurine is a remarkable metabolite concerning hypertension because the metabolite attenuates elevations in the blood pressure of hypertensive patients as well as SHR, although the exact mechanisms are not totally understood. ${ }^{38}$ To the best of our knowledge, there has been no report comparing the urinary level of taurine between the SHR and WKY, except for our previous one. ${ }^{7}$ The excretion of taurine was less in the SHR in the developmental phase of hypertension, and thus the observed changes most likely participate in the onset and development of hypertension in the SHR. It is uncertain whether the hypotaurinuria in the SHR results from the decreased production or the enhanced tubular reabsorption.

Creatinine is an indicator of glomerular filtration rates, and exclusively formed from creatine in the body. Both creatinine and creatine were excreted more in the SHR at 4 weeks of age than in the age-matched WKY. It has been reported that there is no difference in glomerular filtration rates between the SHR and WKY at ages from 2 to 10 weeks. ${ }^{39}$ Thus, it seems reasonable to assume that the increase in creatinine excretion is due to the increase in creatine levels in the body. In fact, there was a moderate correlation $(r=0.550)$ between the urinary levels of the two metabolites in the 4 -week-old rats. The possible reasons for the increased creatine are the enhancement of creatine formation in the liver or the injured skeletal muscle.

The above metabolic changes and the underlying pathophysiological mechanisms in the SHR after the onset of hypertension may be, at least partially, attributable to the elevation of blood pressures rather than the genetic factors. This is a significant point because the changes caused by hypertension are associated with the hypertension-related complications. This issue can be investigated by lowering the blood pressures using antihypertensive drugs, although such drugs may have direct effects on the metabolism irrespective of the hypotensive activity. Such mechanistic investigations would be addressed in the subsequent studies.

The present NMR-based metabonomic study revealed metabolic changes characteristic of genetically hypertensive rats at various ages, covering the prehypertensive period and the period of rapidly rising blood pressure and of sustained hypertension. From the PCA and the quantitative evaluation based on peak heights, the highlighted metabolites and regions were citrate, $\alpha$-ketoglutarate, succinate, hippurate, phenylacetylglycine, $p$-cresol glucuronide, creatine, taurine, mediumchain dicarboxylates (tentative), unknown $(\delta$ 3.11) and the regions at $3.60,3.64,3.68$, and 3.88 p.p.m. The decrease in TCA cycle intermediates, particularly citrate, supported the occurrence of metabolic acidosis in the SHR in the prehypertensive stage as well as in the developing phase of hypertension, which may result from an impairment of the renal energy metabolism. The changes in hippurate, $p$-cresol glucuronide and phenylacetylglycine suggested altered intestinal microflora in the SHR, which were not due to diet but to the genetic and metabolic factors characteristic of the hypertensive strain. Further studies are required to elucidate the pathophysiological significance of other metabolic changes observed in the onset and development of hypertension in the SHR. This study demonstrated that ${ }^{1} \mathrm{H}$-NMR-based urinary metabonomics of the SHR is a useful approach to provide new metabolic information related to hypertension.

\section{CONFLICT OF INTEREST}

The authors declare no conflict of interest.

1 Messerli FH, Williams B, Ritz E. Essential hypertension. Lancet 2007; 370 : 591-603.

2 Johnson RJ, Feig DI, Nakagawa T, Sanchez-Lozada LG, Rodriguez-Iturbe B. Pathogenesis of essential hypertension: historical paradigms and modern insights. J Hypertens 2008; 26: 381-391.

3 Saavedra JM. Opportunities and limitations of genetic analysis of hypertensive rat strains. J Hypertens 2009; 27: 1129-1133.

4 Okamoto K, Aoki K. Development of a strain of spontaneously hypertensive rats. Jpn Circ J 1963; 27: 282-293.

5 Pravenec M, Kurtz TW. Recent advances in genetics of the spontaneously hypertensive rat. Curr Hypertens Rep 2010; 12: 5-9.

6 Westerhuis JA, Van Velzen EJJ, Hoefsloot HCJ, Smilde AK. Multivariate paired data analysis: multilevel PLSDA versus OPLSDA. Metabolomics 2010; 6: 119-128.

7 Akira K, Masu S, Imachi M, Mitome H, Hashimoto M, Hashimoto T. ${ }^{1} \mathrm{H}$ NMR-based metabonomic analysis of urine from young spontaneously hypertensive rats. J Pharm Biomed Anal 2008; 46: 550-556.

8 Baumann M, Van Essen H, Hermans JJR, Smits JFM, Struijker-Boudier HAJ. Functional and structural postglomerular alterations in the kidney of prehypertensive spontaneously hypertensive rats. Clin Exp Hypertens 2004; 26: 663-672.

9 Fukuda S, Tsuchikura S, lida H. Age-related changes in blood pressure, hematological values, concentrations of serum biochemical constituents and weights of organs in the SHR/Izm, SHRSP/Izm and WKY/Izm. Exp Anim 2004; 53: 67-72.

10 Lu Y, A J, Wang G, Hao H, Huang Q, Yan B, Zha W, Gu S, Ren H, Zhang Y, Fan X, Zhang M, Hao K. Gas chromatography/time-of-flight mass spectrometry based metabonomic approach to differentiating hypertension- and age-related metabolic variation in spontaneously hypertensive rats. Rapid Commun Mass Spectrom 2008; 22: 2882-2888.

11 Nicholson JK, Foxall PJ, Spraul M, Farrant RD, Lindon JC. $750 \mathrm{MHz}{ }^{1} \mathrm{H}$ and ${ }^{1} \mathrm{H}-{ }^{13} \mathrm{C}$ NMR spectroscopy of human blood plasma. Anal Chem 1995; 67: 793-811.

12 Lindon JC, Nicholson JK. Biofluids studied by NMR. In Lindon JC, Tranter GE, Holmes JL (eds) Encyclopedia of Spectroscopy and Spectrometry Vol. 1. Academic Press: London, 2000, pp 98-116.

13 Bollard ME, Holmes E, Lindon JC, Mitchell SC, Branstetter D, Zhang W, Nicholson JK. Investigations into biochemical changes due to diurnal variation and estrus cycle in female rats using high-resolution ${ }^{1} \mathrm{H}$ NMR spectroscopy of urine and pattern recognition. Anal Biochem 2001; 295: 194-202.

14 Mortishire-Smith RJ, Skiles GL, Lawrence JW, Spence S, Nicholls AW, Johnson BA Nicholson JK. Use of metabonomics to identify impaired fatty acid metabolism as the mechanism of a drug-Induced toxicity. Chem Res Toxicol 2004; 17: 165-173.

15 Bell JD, Sadler PJ, Morris VC, Levander OA. Effect of aging and diet on proton NMR spectra of rat urine. Magn Reson Med 1991; 17: 414-422.

16 Zuppi C, Messana I, Forni F, Rossi C, Pennacchietti L, Farrari F, Giardina B. ${ }^{1} \mathrm{H}$ NMR spectra of normal urines: reference ranges of the major metabolites. Clin Chim Acta 1997; 265: 85-97.

17 Bollard ME, Stranley EG, Lindon JC, Nicholson JK, Holmes E. NMR-based metabonomic approaches for evaluating physiological influences on biofluid composition. NMR Biomed 2005; 18: 143-162.

18 Bohus E, Coen M, Keun HC, Ebbels TMD, Beckonert O, Lindon JC, Holmes E, Noszal B, Nisholson JK. Temporal metabonomic modeling of L-arginine-induced exocrine pancreatitis. J Proteome Res 2008; 7: 4435-4445.

19 Wang Y, Shu-Hua X, Xue J, Singer BH, Utzinger J, Holmes E. Systems metabolic effects of a Necator americanus infection in Syrian hamster. J Proteome Res 2009; 8 : 5442-5450. 
20 Akira K, Mitome H, Imachi M, Shida Y, Miyaoka H, Hashimoto T. LC-NMR identification of a novel taurine-related metabolite observed in ${ }^{1} \mathrm{H}$ NMR-based metabonomics of genetically hypertensive rats. J Pharm Biomed Anal 2010; 51: 1091-1096.

21 Akira K, Imachi M, Hashimoto T. Investigations into biochemical changes of genetic hypertensive rats using ${ }^{1} \mathrm{H}$ nuclear magnetic resonance-based metabonomics. Hypertens Res 2005; 28: 425-430.

22 Williams RE, Lenz EM, Lowden JS, Rantalainen M, Wilson ID. The metabonomics of aging and development in the rat: an investigation into the effect of age on the profile of endogenous metabolites in the urine of male rats using ${ }^{1} \mathrm{H}$ NMR and HPLC-TOF MS. Mol BioSyst 2005; 1: 166-175.

23 Craig A, Cloarec 0 , Holmes E, Nicholson JK, Lindon JC. Scaling and normalization effects in NMR spectroscopic metabonomic data sets. Anal Chem 2006; 78: 2262-2267.

24 Debska-Slizien A, Ho P, Drangova R, Baines AD. Endogenous dopamine regulates phosphate reabsorption but not NaK-ATPase in spontaneously hypertensive rat kidneys. J Am Soc Nephrol 1994; 5: 1125-1132.

25 Taylor EN, Mount DB, Forman JP, Curhan GC. Association of prevalent hypertension with 24-hour urinary excretion of calcium, citrate, and other factors. Am J Kid Dis 2006; 47: 780-789.

26 Losito A, Nunzi EG, Covarelli C, Nunzi E, Ferrara G. Increased acid excretion in kidney stone formers with essential hypertension. Nephrol Dial Transplant 2009; 24: 137-141.

27 Melnick JZ, Srere PA, Elshourbagy NA, Moe OW, Preisig PA, Alpern RJ. Adenosine triphosphate citrate lyase mediates hypocitraturia in rats. J Clin Invest 1996; 98: 2381-2387.

28 Lucas PA, Lacour B, McCarron DA, Drueke T. Disturbance of acid-base balance in the young spontaneously hypertensive rat. Clin Sci 1987; 73: 211-215.
29 Sharma AM, Distler A. Acid-base abnormalities in hypertension. Am J Med Sci 1994; 307(sppl. 1): S112-S115.

$30 \mathrm{Hamm}$ LL. Renal handling of citrate. Kid Int 1990; 38: 728-735.

31 Pajor AM. Sodium-coupled transporters for Krebs cycle intermediates. Annu Rev Physiol 1999; 61: 663-682.

32 Nissim I, Nissim I, Yudkoff M. Adaptation of renal tricarboxylic acid cycle metabolism to various acid-base states: study with $\left[3-{ }^{13} \mathrm{C}, 5-{ }^{15} \mathrm{~N}\right] \mathrm{glutamine}$. Miner Electrolyte Metab 1991; 17: 21-31.

33 Melnick JZ, Preisig PA, Moe OW, Srere P, Alpern RJ. Renal cortical mitochondrial aconitase is regulated in hypo- and hypercitraturia. Kid Int 1998; 54: 160-165.

34 Hamm LL, Hering-Smith KS. Pivotal role of the kidney in hypertension. Am J Med Sci 2010; 340: 30-32.

35 Goodwin BL, Ruthven CR, Sandler M. Gut flora and the origin of some urinary aromatic phenolic compounds. Biochem Pharmacol 1994; 47: 2294-2297.

36 Holmes E, Loo RL, Stamler J, Bictash M, Yap IKS, Chan Q, Ebbels T, De Lorio M, Brown IJ, Veselkov KA, Daviglus ML, Kesteloot H, Ueshima H, Zhao L, Nicholson JK, Elliott P. Human metabolic phenotype diversity and its association with diet and blood pressure. Nature 2008; 453: 396-400.

37 Nicholson JK, Holmes E, Lindon JC, Wilson ID. The challenges of modeling mammalian biocomplexity. Nat Biotech 2004; 22: 1268-1274.

38 Militante JD, Lombardini JB. Treatment of hypertension with oral taurine: experimental and clinical studies. Amino Acids 2002; 23: 381-393.

39 Christiansen REF, Roald AB, Tenstad O, Iversen BM. Renal hemodynamics during development of hypertension in young spontaneously hypertensive rats. Kid Blood Press Res 2002; 25: 322-328. 